\title{
Prognostic value of the location of submucosal uterine leiomyomas in infertility
}

\author{
GEORGE-ALEXANDRU ROȘU ${ }^{1,2}$, CRÎNGU ANTONIU IONESCU ${ }^{1,2}$, FLORIN DANIEL CĂLIN $^{1,2}$, \\ MIHAI DIMITRIU ${ }^{1,2}$, LIANA PLES $^{1,3}$, ALEXANDRA MATEI $^{1,2}$ and DAN-BOGDAN NAVOLAN ${ }^{4,5}$ \\ ${ }^{1}$ Department of Obstetrics and Gynecology, 'Carol Davila' University of Medicine and Pharmacy, 050474 Bucharest; \\ ${ }^{2}$ Department of Obstetrics and Gynecology, 'Sf. Pantelimon' Clinical Emergency Hospital, 021659 Bucharest; \\ ${ }^{3}$ Department of Obstetrics, 'Bucur' Maternity, 'Sf. Ioan' Clinical Emergency Hospital, 040294 Bucharest; \\ ${ }^{4}$ Department of Obstetrics and Gynecology, 'Victor Babes' University of Medicine and Pharmacy of Timisoara, \\ 300041 Timisoara; ${ }^{5}$ Department of Maternal-Fetal Medicine, 'Dr. Dumitru Popescu' Obstetrics and \\ Gynecology Hospital, 300172 Timisora, Romania
}

Received August 6, 2021; Accepted September 7, 2021

DOI: $10.3892 /$ etm.2021.10917

\begin{abstract}
Submucosal fibroid location and size are predictive factors of impaired fertility. Submucosal fibroids cause infertility through several mechanisms including distortion of the endometrial cavity, increased uterine contractility, local inflammation and remodeling of the endometrial blood supply. This is a monocentric, retrospective, cross-sectional study, conducted in the Department of Obstetrics and Gynecology of 'Sf. Pantelimon' Clinical Emergency Hospital, analyzing patients from a 5-year period (January 2015-December 2019). In the present study, the relationship between different characteristics of the submucosal fibroids (among others, location and dimensions) and fertility (birth rates, early pregnancy loss rates) were investigated. This study identified that submucosal and intramural fibroids are risk factors for reduced birth rate compared with subserosal fibroids $(\mathrm{P}=0.02, \mathrm{RR}=2.58,95 \% \mathrm{CI}$ 1.03-6.47; $\mathrm{P}=0.005, \mathrm{RR}=1.18,95 \%$ CI 1.02-1.35, respectively). In addition, $\mathrm{G} 2$ leiomyomas are risk factors for low birth rate compared with $\mathrm{G} 0$ and $\mathrm{G} 1$ fibroids $(\mathrm{P}=0.01, \mathrm{RR}=1.95,95 \% \mathrm{CI}$ 1.05-3.60). Moreover, the presence of a subserosal fibroid was associated with an increased early pregnancy loss rate $(\mathrm{P}=0.01$, $\mathrm{RR}=2.14,95 \% \mathrm{CI}$ 1.05-4.35). In conclusion, the location and degree of uterine cavity distortion are important factors that alter the normal development of a pregnancy and the birth rate.
\end{abstract}

Correspondence to: Professor Crîngu Antoniu Ionescu, Department of Obstetrics and Gynecology, 'Sf. Pantelimon' Clinical Emergency Hospital, 340-342 Șoseaua Pantelimon Boulevard, 021659 Bucharest, Romania

E-mail: antoniuginec@yahoo.com

Key words: uterine fibroid, submucosal leiomyoma, reproductive medicine, infertility, pregnancy, clinical diagnosis

\section{Introduction}

Uterine leiomyomas are the most common benign gynecological tumor in fertile age women, affecting up to $60-80 \%$ of the females under 50 years of age $(1,2)$. These tumors are a unique cause of infertility in 1-3\% of cases of infertile women (2). Many studies and reviews emphasize the detrimental effects that fibroids have on fertility, those consequences being based on numerous mechanisms (3-6).

The mechanical effect is produced by the distortion of the endometrial cavity, caused especially by the submucosal fibroids (5), although some studies have identified a negative correlation between intramural fibroids and fertility, especially in cases of patients who follow assisted reproductive techniques (7-9), induced by the alteration of the myometrial anatomy, albeit this may not be the sole mechanism responsible (3). Additionally, the size of the fibroid may influence the degree of reshaping of the uterine cavity; larger submucosal fibroids, with a diameter greater than $4 \mathrm{~cm}$, have been found to be responsible for the alteration of the implantation process, in particular in cases of artificial fertilization $(2,10,11)$.

The location of leiomyomas related to the endometrial cavity also contributes to the alteration of the uterine milieu regarding the fecundation and implantation of the blastocyst, this aspect being visible especially in cases of failed assisted reproductive techniques $(2,11,12)$. Leiomyomas located on the anterior wall influence early pregnancy, increasing the abortion rate (11). Additionally, fibroids situated in the fundic wall have been identified to increase preterm birth rates (11).

The local functional alterations observed in cases of uterine leiomyomas, especially submucosal and intramural fibroids, are represented by increased uterine contractility that interfere with embryo transfer/gamete migration and embryo implantation and remodeling of the endometrial blood supply that leads to glandular atrophy, chronic inflammatory reaction and endometrial ulceration, finally resulting in implantation failure or early pregnancy loss $(4,7,13)$. 
The alteration of gene expression due to overstretch of the uterine walls, caused in particular by intramural fibroids or fibroids with an important intramural component such as G2 fibroids, decreases endometrial receptivity, as observed during the window of implantation $(1,14)$. The homeobox A $(H O X A)$ genes, which are expressed at the level of the female reproductive tract and are involved in implantation, in particular the HOXA-10 and HOXA-11 genes, have a reduced expression in patients that present with infertility and uterine leiomyomas, and are also related to the repeated implantation failure in patients who follow assisted reproductive technologies $(1,14,15)$.

The biochemical changes that appear in the presence of uterine leiomyomas are numerous and diverse. Low levels of interleukin (IL)-10 and glycodelin in the mid-luteal uterine flushings in patients with leiomyomas have been previously reported $(13,16)$. These cytokines are involved in the implantation process during the window of implantation, and lower levels could alter this mechanism (1). Tumor necrosis factor (TNF)- $\alpha$, an immunomodulatory cytokine that has a role in fibroid cell apoptosis, being found in higher levels in uterine flushings of patients with fibroids and in serum analysis, is also involved in the preimplantation development of the embryo, trophoblast invasion regulation and immunological pregnancy loss $(17,18)$. Transforming growth factor (TGF)- $\beta$, a paracrine molecule, secreted and overexpressed in uterine fibroids, is connected with the disturbance of endometrial receptivity and decidualization, leading to early pregnancy loss $(19,20)$. Fibroblast growth factor (FGF), a proinflammatory mediator with elevated serum values in patients with uterine leiomyomas, is also linked to infertility and reproductive failure $(18,21)$.

The main diagnostic methods employed in the diagnosis of uterine leiomyomas are represented by $2 \mathrm{D}$ transvaginal ultrasonography, hysteroscopy (for submucosal fibroids) (22), computed tomography or magnetic resonance imaging, none of which are considered 'gold standard' for this diagnosis, and each of which have their advantages and limitations. In the diagnosis of submucosal leiomyoma, two-dimensional saline contrast sonohysterography has been identified to be a highly sensitive diagnostic method for this pathology, having the potential to become the first-line diagnostic technique (23).

The main objective of the study was to evaluate the incidence of submucosal leiomyomas and the association between their location in infertility compared with intramural fibroids in a single center, analyzing data from a 5-year time frame.

\section{Patients and methods}

Subjects. A monocentric, retrospective, longitudinal study was conducted, analyzing the 931 cases admitted to the Department of Obstetrics and Gynecology from 'Sf. Pantelimon' Clinical Emergency Hospital from Bucharest, Romania, during a 5-year timeframe, from January 2015 to December 2019 for the diagnosis of uterine leiomyoma. Although the fibroids could have changed their characteristics during the period between last obstetric event (birth or miscarriage) of a particular patient and the time of hysterectomy, all the patients presented leiomyomas during their previous fertile years and during their prior pregnancies and miscarriages. The investigation of past miscarriages, premature births, and periods of infertility in hysterectomized patients was based on previous medical records and interviews prior to surgery. In this sample, other possible infertility factors (e.g., fallopian tubes, male infertility) were excluded on the basis of previous medical records. In each case the relationship between submucosal or intramural fibroids characteristics and the fertility rate, abortion rate and preterm birth rate was analyzed. An analysis was conducted on the subgroups based on the location of the submucosal fibroid in relation to the endometrial cavity and the pregnancy and abortion rates in these subgroups.

The patients provided their informed consent for the use of their data in studies and upon admission to the hospital. The study received ethical approval from the Ethics Commission of the 'Sf. Pantelimon' Clinical Emergency Hospital (protocol code 1200/18.01.2021), the study being conducted according to the guidelines of the Declaration of Helsinki.

Statistical analysis. Statistical analysis was performed using the Vassar Stats website (http://vassarstats.net/) and the Minitab Statistical Software (version 19) (https://www. minitab.com/en-us/products/minitab/). The Mann-Whitney $\mathrm{U}$ test was used to compare quantitative variables while the Chi-square test was used to compare qualitative variables. $\mathrm{P}<0.05$ was considered to indicate statistical significance.

\section{Results}

This monocentric study analyzed retrospectively all admissions to the Department of Obstetrics and Gynecology from the 'Sf. Pantelimon' Clinical Emergency Hospital from 1st January, 2015 until 31st December, 2019, and search for all cases that had as main or secondary diagnoses 'uterine leiomyoma'. The analyzed cases included in the study were represented by patients who had performed hysterectomies for various benign diagnoses, and for which the histopathological examination had identified and described in detail the localization and characteristics of the fibroids. For these patients, all the elements related to their fertility were taken into evaluation including number of births, number of abortions, number of preterm births, the type of infertility (primary or secondary) and the number of years of infertility.

During the 5-year time frame, 931 patients were admitted to the Department of Obstetrics and Gynecology from 'Sf. Pantelimon' Clinical Emergency Hospital for various symptoms (pelvic pain, methroragies, dyspareunia) and were diagnosed with a benign condition for which a hysterectomy was performed. From these cases, 292 histopathological specimens were identified to have one or more leiomyomas. This group was further subdivided into four subcategories: patients that had only submucosal fibroids $(18 / 292 ; 6.16 \%)$, patients only with intramural fibroids $(167 / 292 ; 57.19 \%)$, patients who have had simultaneous submucosal and intramural fibroids $(85 / 292$; $29.10 \%$ ) and patients that presented only subserosal fibroids $(22 / 292,7.53 \%)$, this last group being used as a 'internal control group' to perform the statistical analysis, knowing from previous studies that this type of fibroid has a minor or neglectable influence in fertility (Table I). The patients with submucosal fibroids, regardless of the association with other fibroids, were analyzed separately, by subdividing this category in three subgroups, based on the FIGO classification system: G0 (14/103; 13.59\%), G1 (37/103; 35.92\%) and G2 (52/103; 50.48\%) (Table II). 
Table I. Patients with leiomyomas identified using the histopathological specimen obtained after hysterectomies performed, between January 2015 and December 2019, for benign indications.

\begin{tabular}{|c|c|c|c|c|}
\hline Variables & SM & IM & $\begin{array}{l}\text { SM and IM } \\
\text { (simultaneous) }\end{array}$ & SS \\
\hline No. of patients $(\%)$ & $18(6.16 \%)$ & $167(57.19 \%)$ & $85(29.10 \%)$ & $22(7.53 \%)$ \\
\hline \multicolumn{5}{|l|}{ Age at the moment of the hysterectomy (years) } \\
\hline Min/Max & $38 / 63$ & $29 / 71$ & $34 / 69$ & $32 / 66$ \\
\hline Mean & 47 & 46.29 & 47.62 & 45.90 \\
\hline Median & 46 & 45 & 47 & 44 \\
\hline \multicolumn{5}{|l|}{ BMI } \\
\hline Min/Max & $22 / 41$ & $19 / 45$ & $19 / 41$ & $20 / 40$ \\
\hline Mean & 26.22 & 27.13 & 28 & 25.45 \\
\hline Median & 25.5 & 26 & 26 & 25.5 \\
\hline \multicolumn{5}{|l|}{ No. of fibroids } \\
\hline Min/Max & $1 / 3$ & $1 / 6$ & $1 / 5$ & $1 / 2$ \\
\hline Mean & 1.22 & 1.93 & 2.06 & 1.13 \\
\hline Median & 1 & 1 & 2 & 1 \\
\hline \multicolumn{5}{|l|}{ Maximum diameter of the largest fibroid (cm) } \\
\hline $\operatorname{Min} / \operatorname{Max}$ & $1 / 5$ & $2 / 16$ & $1 / 14$ & \\
\hline Mean & 2.72 & 5.59 & 4.44 & N/A \\
\hline Median & 3 & 5 & 4 & \\
\hline Patients with primary infertility, $\mathrm{n}(\%)$ & 0 & $2(1.19 \%)$ & $1(1.17 \%)$ & $4(18.27 \%)$ \\
\hline Patients with secondary infertility, n (\%) & $5(27.7 \%)$ & $35(20.95 \%)$ & $24(28.23 \%)$ & $4(18.27 \%)$ \\
\hline \multicolumn{5}{|l|}{$\begin{array}{l}\text { Years of infertility (n) (in cases of } \\
\text { infertile patients) }\end{array}$} \\
\hline Min/Max & $3 / 5$ & $2 / 12$ & $3 / 13$ & $3 / 10$ \\
\hline Mean & 3.6 & 5.43 & 5.13 & 6.25 \\
\hline Median & 3 & 5 & 4,5 & 6 \\
\hline \multicolumn{5}{|l|}{ Births (n) } \\
\hline Min/Max & $0 / 3$ & $0 / 5$ & $0 / 4$ & $0 / 4$ \\
\hline Mean & 2.16 & 2.19 & 2.17 & 1.68 \\
\hline Median & 2 & 2 & 2 & 2 \\
\hline \multicolumn{5}{|l|}{ Abortions (n) } \\
\hline Min/Max & $0 / 3$ & $0 / 3$ & $0 / 4$ & $0 / 4$ \\
\hline Mean & 0.47 & 0.32 & 0.44 & 0.27 \\
\hline Median & 0 & 0 & 0 & 0 \\
\hline \multicolumn{5}{|l|}{ PTB, n/total (\%) } \\
\hline $\begin{array}{l}\text { PTB from total number of births in } \\
\text { that category }\end{array}$ & $1 / 39(2.56 \%)$ & $27 / 367(7.35 \%)$ & $13 / 185(7.02 \%)$ & $2 / 37(5.40 \%)$ \\
\hline $\begin{array}{l}\text { Patients with PTB from total number } \\
\text { of patients in that category }\end{array}$ & $1 / 18(5.55 \%)$ & $23 / 167(16.78 \%)$ & $12 / 85(14.11 \%)$ & $2 / 22(9.09 \%)$ \\
\hline
\end{tabular}

SM, submucosal fibroids; IM, intramural fibroids; SS, subserosal fibroids; BMI, body mass index; PTB, preterm births.

Fibroid characteristics. Comparing the four subgroups, we observed that the largest number of patients presented only intramural fibroids $(167 / 292 ; 57.19 \%)$, followed by the one that had both intramural and submucosal leiomyomas $(85 / 292$; $29.10 \%$ ). The smallest number of patients was included in the first subgroup, patients that presented only submucosal fibroids $(18 / 292 ; 6.16 \%)$. However, when considering all the cases that presented with at least one submucosal fibroid, as the only type of fibroid or in association with other types (especially with intramural fibroids), the number of patients that had submucosal fibroids were 103 of 292 patients (35.27\%), representing one-third of the cases that presented with uterine leiomyomas and had a hysterectomy performed for benign indications. When we analyzed the number of fibroids per patient in each category, we found that the largest number of fibroids was identified in the cases that presented intramural fibroids (up 
Table II. Patients with submucosal fibroids identified based on the histopathological specimen (subgroup from the hysterectomy group).

\begin{tabular}{|c|c|c|c|c|}
\hline Variables & All SM & SM-G0 & SM-G1 & SM-G2 \\
\hline No. of patients, n (\%) & $103(100 \%)$ & $14(13.59 \%)$ & $37(35.92 \%)$ & $52(50.48 \%)$ \\
\hline \multicolumn{5}{|l|}{ Age at the moment of the hysterectomy (years) } \\
\hline $\operatorname{Min} / \operatorname{Max}$ & $34 / 69$ & $41 / 56$ & $38 / 68$ & $34 / 69$ \\
\hline Mean & 47.51 & 47 & 48.54 & 46.92 \\
\hline Median & 47 & 46.5 & 48 & 46.5 \\
\hline \multicolumn{5}{|l|}{ BMI } \\
\hline Min/Max & $19 / 41$ & $21 / 38$ & $20 / 41$ & $19 / 41$ \\
\hline Mean & 27.68 & 26.92 & 28.35 & 27.42 \\
\hline Median & 26 & 25 & 27 & 26 \\
\hline \multicolumn{5}{|l|}{$\begin{array}{l}\text { Location of the largest fibroid (related to } \\
\text { uterine walls), n (\%) }\end{array}$} \\
\hline Anterior & $51(49.51 \%)$ & $7(50 \%)$ & $15(40.54 \%)$ & $29(55.76 \%)$ \\
\hline Posterior & $38(36.89 \%)$ & $5(34.71 \%)$ & $18(48.64 \%)$ & $15(28.84 \%)$ \\
\hline Fundal & $14(13.59 \%)$ & $2(14.28 \%)$ & $4(10.81 \%)$ & $8(15.38 \%)$ \\
\hline \multicolumn{5}{|l|}{ Maximum diameter of the largest fibroid (cm) } \\
\hline $\operatorname{Min} / \operatorname{Max}$ & $1 / 6$ & $1 / 6$ & $1 / 6$ & $1 / 6$ \\
\hline Mean & 3.06 & 2.85 & 3.05 & 3.13 \\
\hline Median & 3 & 3 & 3 & 3 \\
\hline Patients with primary infertility, n (\%) & $1(0.97 \%)$ & $1(7.14 \%)$ & 0 & 0 \\
\hline Patients with secondary infertility, n (\%) & $30(29.12 \%)$ & $5(35.71 \%)$ & $8(21.62 \%)$ & $16(30.76 \%)$ \\
\hline \multicolumn{5}{|l|}{$\begin{array}{l}\text { Years of infertility (in cases of infertile } \\
\text { patients) (n) }\end{array}$} \\
\hline $\operatorname{Min} / \operatorname{Max}$ & $3 / 13$ & $3 / 13$ & $3 / 7$ & $3 / 12$ \\
\hline Mean & 5 & 6.6 & 4.37 & 4.85 \\
\hline Median & 4 & 6 & 4 & 4 \\
\hline \multicolumn{5}{|l|}{ Births (n) } \\
\hline Min/Max & $0 / 4$ & $0 / 3$ & $1 / 4$ & $1 / 4$ \\
\hline Mean & 2.17 & 2.21 & 2.35 & 2.02 \\
\hline Median & 2 & 2 & 2 & 2 \\
\hline \multicolumn{5}{|l|}{ Abortions (n) } \\
\hline Min/Max & $0 / 4$ & $0 / 2$ & $0 / 3$ & $0 / 4$ \\
\hline Mean & 0.45 & 0.42 & 0.39 & 0.5 \\
\hline Median & 0 & 0 & 0 & 0 \\
\hline \multicolumn{5}{|l|}{ PTBs, n/total (\%) } \\
\hline $\begin{array}{l}\text { Number of PTB from total number of births } \\
\text { in that category }\end{array}$ & $14 / 224(6.25 \%)$ & $0 / 31$ & 8/87 (9.19\%) & $6 / 106(5.66 \%)$ \\
\hline $\begin{array}{l}\text { Number of patients with PTB from total } \\
\text { number of patients in that category }\end{array}$ & $13 / 103(12.62 \%)$ & $0 / 14$ & $7 / 37(18.91 \%)$ & $6 / 52(11.53 \%)$ \\
\hline
\end{tabular}

SM, submucosal fibroids; BMI, body mass index; PTB, preterm births.

to six fibroids), although the average number of fibroids per patient was highest in the subgroup where submucosal and intramural fibroids were found in association (mean 2.06; median value 2). The largest fibroids were also identified in the intramural category, with the largest diameter between 2 and $16 \mathrm{~cm}$ (mean $5.59 \mathrm{~cm}$; median value $5 \mathrm{~cm}$ ).

Analyzing the distribution of submucosal fibroids based on the FIGO leiomyoma subclassification system, 14 of
103 patients had a G0 leiomyoma (13.59\%), 37 patients $(35.92 \%)$ presented at least one G1 fibroid, and 52 patients $(50.48 \%)$ had a G2 leiomyoma, this last group being the largest. Evaluating the location of the submucosal fibroids in relation with uterine walls, the most frequent location was at the level of the anterior wall $(49.51 \%)$, followed by the posterior wall $(36.89 \%)$ and least at the level of uterine fundus $(13.59 \%)$. No statistically significant differences between the submucosal 
fibroids subgroup and intramural fibroids were identified when the maximum diameter of the largest fibroid was analyzed ( $\mathrm{P}=0.07, \mathrm{RR}=1.35,95 \% \mathrm{CI}: 0.97-1.88)$.

Body mass index. Compared with normal weight patients, overweight and obese patients did not present any statistically significant difference when leiomyoma locations inside the uterine wall were analyzed (subserosal vs. submucosal fibroids: $\mathrm{P}<0.60, \mathrm{RR}=0.97$ 95\% CI: 0.49-1.94; subserosal vs. intramural fibroids: $\mathrm{P}<0.39, \mathrm{RR}=0.97,95 \% \mathrm{CI}$ : 0.87-1.08; subserosal vs. simultaneous submucosal and intramural fibroids: $\mathrm{P}<0.10, \mathrm{RR}=0.84,95 \% \mathrm{CI}$ : 0.66-1.07).

Infertility analysis. The first element analyzed was represented by the number of patients with infertility. In the entire group, there were 7 patients with primary infertility and 68 patients with secondary infertility. The largest number of patients with primary infertility was in the subserosal subgroup (4 of 7 patients with primary infertility; $57.14 \%$ ), most probably the cause of infertility being other than the subserosal leiomyoma. In cases of secondary infertility, the highest number of patients was in the intramural leiomyoma subgroup (35 of 68 patients with secondary infertility, representing $51.47 \%$ of these cases). The number of years of infertility in the cases of infertile patients (regardless the type of infertility) largely varied from 2 to 13 years, with the largest mean in the subserosal subgroup (6.25 years), followed by the intramural subgroup with 5.43 years

In the distinct analysis of the submucosal fibroids, there was one patient with primary infertility and 30 patients with secondary infertility, the highest number being found in the G2 subgroup (16/30 patients; 53.33\%).

Fertility analysis. The birth rate in patients with uterine leiomyoma was around 2 births per patient, with the lowest mean for the subgroup with subserosal fibroids (1.68), although the median value for the subcategories was 2 births/patient. When we compared the birth rate between subgroups (divided using the fibroid location in the uterine wall and its relationship with the uterine cavity), applying the mean value as the cut-off value, we identified risk factors that reduced the birth rate, altering the pregnancy outcome. In our sample, the submucosal and intramural fibroids were identified as risk factors for this outcome compared with subserosal leiomyomas $(\mathrm{P}=0.02$, $\mathrm{RR}=2.58,95 \% \mathrm{CI}: 1.03-6.47 ; \mathrm{P}=0.005, \mathrm{RR}=1.18,95 \% \mathrm{CI}$ : $1.02-1.35$, respectively). In addition, when we compared the birth rates between patients with simultaneous submucosal and intramural leiomyomas and patients with subserosal fibroids, we also identified a statistical difference between the two groups, the presence of simultaneous submucosal and intramural fibroids representing a risk factor for the birth rate $(\mathrm{P}=0.005, \mathrm{RR}=1.36,95 \% \mathrm{CI}: 1.05-1.77)$. In the submucosal cluster, we determined a statistical difference between the G2 fibroids compared with the G0 and G1 fibroids taken together $(\mathrm{P}=0.01, \mathrm{RR}=1.95,95 \% \mathrm{CI}$ : $1.05-3.60)$.

Assessing the importance of submucosal fibroid location in relation to one of the uterine walls (anterior, posterior and fundic), in this sample we identified as risk factors the location of the fibroid at the level of the anterior or posterior wall compared with the fundal location $(\mathrm{P}=0.0001, \mathrm{R}=1.76$,
95\% CI: 1.22-2.53; $\mathrm{P}=0.0006, \mathrm{RR}=1.85,95 \% \mathrm{CI}: 1.22-2.80$, respectively).

Evaluating the preterm birth rate in every one of the subgroups of the hysterectomy batch we did not find any statistical difference between the four categories, although we observed a larger proportion of preterm births in the intramural subcategory, in both instances, when we compared the percentage of preterm births to the total number of births in that category (27 preterm births out of 367 births in the intramural-only category, representing $7.35 \% ; \mathrm{P}=0.38, \mathrm{R}=1.58$, 95\% CI: 0.39-6.28) and the number of patients who had at least one preterm birth from the total number of patients in that category (23 patients out of 167 patients in the intramural-only category, representing $16.78 \%$ ). This is also the case, when the submucosal cluster was analyzed: there were no statistical differences between the subgroups, but a higher percentage of preterm births was identified in the G1 subgroup compared with the G2 subcategory $(9.19 \%$ vs. $5.66 \%$, when the number of preterm births was reported to the total number of births in that category; $\mathrm{P}=0.25, \mathrm{RR}=1.63,95 \% \mathrm{CI}: 0.59-4.48$ ), considering the fact that in the G0 subgroup no preterm birth was registered. Comparing the three possible locations of the submucosal fibroids in relation to the uterine walls, i.e., anterior, posterior or fundal location, there was no statistically significant difference (anterior vs. posterior: $\mathrm{P}=0.59, \mathrm{RR}=1.04$, 95\% CI 0.35-3.03; anterior with fundal: $\mathrm{P}=0.44, \mathrm{RR}=1.92$, 95\% CI: $0.25-14.34$; fundal with posterior location: $\mathrm{P}=0.44$, $\mathrm{RR}=1.84$, 95\% CI: 0.23-14.42).

Assessment of the early pregnancy loss (labelled as abortions in Tables I and II) identified statistical differences, although we did not have a clinical explanation for this finding. The subserosal leiomyomas are a risk factor for early pregnancy loss compared with the simultaneous presence of submucosal and intramural fibroids, $(\mathrm{P}=0.01, \mathrm{RR}=2.14,95 \%$ CI: 1.05-4.35) or all submucosal fibroids cases (103 cases), regardless of the association of these leiomyomas with other types of fibroids $(\mathrm{P}=0.01, \mathrm{RR}=2.16,95 \% \mathrm{CI}$ : $1.07-4.50)$. Neither the submucosal fibroids $(\mathrm{P}=0.059, \mathrm{RR}=0.62,95 \% \mathrm{CI}$ : 0.39-1.0007) or intramural fibroids alone $(\mathrm{P}=0.13, \mathrm{R}=0.94$, 95\% CI: 0.88-1.02) had statistically significant influence on the miscarriage rate.

\section{Discussion}

The submucosal and intramural uterine leiomyomas presented a negative influence for the birth rate compared with the subserosal fibroids, in our study. In addition, the simultaneous presence of submucosal and intramural fibroids, compared with subserosal leiomyomas, affect in a greater manner the successful development of a pregnancy until term and the birth rate. These results are similar to the ones reported in the literature, owing these effects to the distortion of endometrial cavity, mechanically affecting the implantation process, in the case of submucosal fibroids $(3-5,11)$, and to myometrial anatomy disarrangement (7-9) and the disruption and remodeling of the endometrial blood supply, causing endometrial ulcerations and inflammation, when non-cavity-distorting intramural leiomyomas are present $(7,12,14)$.

When the body mass index (BMI) was analyzed to evaluate the influence of a higher BMI on the fibroid's location inside the 
uterine wall, no statistically significant difference was identified between patients with normal weight and overweight or obese patients. In addition, we did not find in the literature studies that analyzed this association. This analysis was conducted considering the fact that overweight and obese patients present higher levels of estrogens, hormones that influence the genesis and development of these tumors, and for this reason it was considered that a superior level of estrogens stimulate the development of intramural or submucosal fibroids, rather than subserosal ones. Further studies should be designed to analyze the relationship between weight, body mass index, estrogen levels and the fibroid size and location, to identify whether there is any association between these elements.

In the submucosal fibroids group, the presence of a leiomyoma that has at least $50 \%$ of its mean diameter intramural (type 2 or G2 fibroid in the FIGO leiomyoma subclassification system) presented a statistical significant difference in reducing the birth rate, compared with the other two types of submucosal fibroids (type 0 or G0 fibroids, intracavitary lesion that is attached to the endometrium by a narrow stalk; type 1 or G1 leiomyomas, a leiomyoma that has less than $50 \%$ of its mean diameter intramural). This observation was different from the one reported in an article by Litta et al on the pregnancy outcome after hysteroscopic myomectomy (11). One possible explanation is the greater myometrial disarrangement produced by the $\mathrm{G} 2$ fibroids compared with the two other subtypes of submucosal fibroids. Prospective studies should focus on this difference and the exact cause of this influence on the birth rate.

The location of submucosal fibroids in relation to uterine cavity walls proved to be another risk factor for pregnancy and birth rate. The anterior and posterior location of submucosal leiomyomas are risk factors for pregnancy development until birth, compared with the fundal location. The greater extent of disturbance that the fibroids located on the anterior and posterior wall have on the uterine cavity, compared with the fundal ones, perturb the normal processes of embryo implantation of the zygote. Most studies excluded the location of fibroids in relation to uterine walls in their analysis (3); for this reason it is difficult to correlate this result with the literature. Litta et al found that the miscarriage rate was statistically significant in the anterior location of fibroids and preterm delivery rate was statistically significant in the fundal location of fibroids, conclusions that were not evident in the present study (11).

Of note, subserosal fibroids were associated with a statistically significant higher rate of miscarriages compared with the simultaneous presence of submucosal and intramural fibroids even when the subserosal subgroup was compared with all the cases that presented submucosal fibroids, regardless of the association with other types of fibroids. A possible explanation could be the fact that some subserosal fibroids present at least $50 \%$ of its mean diameter inside the uterine wall (type 5 fibroids in the FIGO leiomyoma subclassification system), and for this reason it alters the myometrial architecture, disturbing the implantation process, as the non-cavity-distorting intramural leiomyomas do. It is unclear why this association occurs and further studies should analyze the effect of subserosal leiomyomas on fertility in a more explicit manner.

The retrospective and monocentric characteristics of this research represent some of the limits of the present study. The retrospective aspect made it difficult to evaluate and characterize in a more specific manner the submucosal leiomyomas, other than the aspect evaluated at the moment of patient admission to our clinic. For example, there were only a few saline contrast hysterosonographies performed to describe more accurately cavity involvement and the exact location of submucosal fibroids in relation to cornual or cervical regions. For this reason, a prospective study should be conducted using a standardized protocol for the evaluation of the fertility characteristics of patients with submucosal fibroids, using also the molecular and biochemical markers proven to be influenced by uterine leiomyomas, which are in relation to endometrial receptivity. Another limitation of our retrospective study was represented by the factors, other than leiomyomas, that could influence the obstetric history and that were partially analyzed, only by taking medical history.

The conclusions of this study are the fact that the location of submucosal fibroids and the degree of uterine cavity distortion are important factors that alter the normal development of a pregnancy and the birth rate, and also that non-cavity-distorting leiomyomas influence the fertility rate and early pregnancy loss.

\section{Acknowledgements}

Not applicable.

\section{Funding}

No funding was received.

\section{Availability of data and materials}

The datasets used and analyzed during the current study are available from the corresponding author on reasonable request.

\section{Authors' contributions}

The conceptualization of this study was performed by GAR and CAI. The methodology for the study was carried out by FDC, MD and LP. The validation of this study was performed by CAI and DBN. The formal analysis was carried out by MD and FDC. The investigation was conducted by FDC, LP, DBN and AM. The resources were ensured by MD and FDC. The data curation was performed by GAR. The writing of the original draft was done by GAR, AM and CAI and the review and editing was performed by CAI and DBN. The visualization of the article was carried out by GAR. The interpretation of the data for this work was carried out by GAR, CAI and MD. The supervision for this study was assured by CAI. All authors have read and approved the final manuscript.

\section{Ethics approval and consent to participate}

This study was performed in line with the principles of the Declaration of Helsinki. Approval was granted by the Ethics Committee of 'Sf. Pantelimon' Clinical Emergency Hospital (1200/18.01.2021). Informed consent was obtained from all individual participants included in the study. 


\section{Patient consent for publication}

Patients signed informed consent regarding publishing their data when they were admitted to the hospital.

\section{Competing interests}

The authors declare that they have no competing interests.

\section{References}

1. Ikhena DE and Bulun SE: Literature review on the role of uterine fibroids in endometrial function. Reprod Sci 25: 635-643, 2018.

2. Levy G, Hill MJ, Beall S, Zarek SM, Segars JH and Catherino WH: Leiomyoma: Genetics, assisted reproduction, pregnancy and therapeutic advances. J Assist Reprod Genet 29: 703-712, 2012.

3. Pritts EA, Parker WH and Olive DL: Fibroids and infertility: An updated systematic review of the evidence. Fertil Steril 91: $1215-1223,2009$

4. Gambadauro P: Dealing with uterine fibroids in reproductive medicine. J Obstet Gynaecol 32: 210-216, 2012.

5. Brady PC, Stanic AK and Styer AK: Uterine fibroids and subfertility: An update on the role of myomectomy. Curr Opin Obstet Gynecol 25: 255-259, 2013.

6. Carranza-Mamane B, Havelock J,Hemmings R and Reproductive Endocrinology and Infertility Committee; Special Contributor: The management of uterine fibroids in women with otherwise unexplained infertility. J Obstet Gynaecol Can 37: 277-285, 2015

7. Zepiridis LI, Grimbizis GF and Tarlatzis BC: Infertility and uterine fibroids. Best Pract Res Clin Obstet Gynaecol 34: 66-73, 2016.

8. Christopoulos G, Vlismas A, Salim R, Islam R, Trew G and Lavery S: Fibroids that do not distort the uterine cavity and IVF success rates: An observational study using extensive matching criteria. BJOG 124: 615-621, 2017.

9. Styer AK, Jin S, Liu D, Wang B, Polotsky AJ, Christianson MS, Vitek W, Engmann L, Hansen K, Wild R, et al: Association of uterine fibroids and pregnancy outcomes after ovarian stimulation- intrauterine insemination for unexplained infertility. Fertil Steril 107: 756-762.e3, 2017.

10. Sarıdoğan E and Sarıdoğan E: Management of fibroids prior to in vitro fertilization/intracytoplasmic sperm injection: A pragmatic approach. J Turk Ger Gynecol Assoc 20: 55-59, 2019.

11. Litta P, Conte L, De Marchi F, Saccardi C and Angioni S: Pregnancy outcome after hysteroscopic myomectomy. Gynecol Endocrinol 30: 149-152, 2014.
12. Sagi-Dain L, Ojha K, Bider D, Levron J, Zinchenko V, Walster S, Sagi S and Dirnfeld M: Pregnancy outcomes in oocyte recipients with fibroids not impinging uterine cavity. Arch Gynecol Obstet 295: 497-502, 2017.

13. Purohit $P$ and Vigneswaran K: Fibroids and Infertility. Curr Obstet Gynecol Rep 5: 81-88, 2016.

14. Unlu C, Celik O, Celik N and Otlu B: Expression of endometrial receptivity genes increase after myomectomy of intramural leiomyomas not distorting the endometrial cavity. Reprod Sci 23: 31-41, 2016.

15. Rackow BW and Taylor HS: Submucosal uterine leiomyomas have a global effect on molecular determinants of endometrial receptivity. Fertil Steril 93: 2027-2034, 2010.

16. Ben-Nagi J, Miell J, Mavrelos D, Naftalin J, Lee C and Jurkovic D: Endometrial implantation factors in women with submucous uterine fibroids. Reprod Biomed Online 21: 610-615, 2010.

17. Demir M, Kalyoncu S, Ince O, Ozkan B, Kelekci S, Saglam G, Sutcu R and Yilmaz B: Endometrial flushing tumor necrosis factor alpha and interleukin 2 levels in women with polycystic ovary syndrome, leiomyoma and endometrioma: Comparison with healthy controls. Geburtshilfe Frauenheilkd 79: 517-523, 2019.

18. Sevostyanova O, Lisovskaya T, Chistyakova G, Kiseleva M, Sevostyanova N, Remizova I and Buev Y: Proinflammatory mediators and reproductive failure in women with uterine fibroids. Gynecol Endocrinol 36: 33-35, 2020.

19. Doherty LF and Taylor HS: Leiomyoma-derived transforming grow th factor- $\beta$ impairs bone morphogenetic protein-2-mediated endometrial receptivity. Fertil Steril 103: 845-852, 2015.

20. Ciarmela P, Islam MS, Reis FM, Gray PC, Bloise E, Petraglia F, Vale $\mathrm{W}$ and Castellucci M: Growth factors and myometrium: Biological effects in uterine fibroid and possible clinical implications. Hum Reprod Update 17: 772-790, 2011.

21. Călin F, Ionescu C, Dimitriu M, Ciobanu AM, Badiu CD, Neacsu A, Socea B and Hudiță D: The impact of uterine fibromatosis on the endometrium and fertility. J Surg Sci: Sept 2020. doi: 10.33695/jss.v7i2.350.

22. Matei A, Ionescu C, Gorun F, Gheorghiu D, Rosu GA, Dan A Sima RM, Furau CG, Ilinca C and Navolan D: Insights on hysteroscopic procedures and their place in Romanian gynecologic practice- the experience of two medical units. Diagnostics (Basel) 10: 281, 2020.

23. Bittencourt CA, Dos Santos Simões R, Bernardo WM, Fuchs LFP, Soares Júnior JM, Pastore AR and Baracat EC: Accuracy of saline contrast sonohysterography in detection of endometrial polyps and submucosal leiomyomas in women of reproductive age with abnormal uterine bleeding: Systematic review and meta-analysis. Ultrasound Obstet Gynecol 50: 32-39, 2017. 